\title{
Renal Tumor-Reactive Autologous Peripheral Blood Lymphocyte
}

National Cancer Institute

\section{Source}

National Cancer Institute. Renal T umor-Reactive Autologous Peripheral Blood

Lymphocyte. NCI Thesaurus. Code C48816.

Peripheral blood lymphocytes (PBL) harvested from the blood of a renal cancer patient and exposed in vitro to renal tumor-associated antigens (TAA). Introducing these renal tumor-reactive autologous peripheral blood lymphocytes back to the same patient target tumor cells expressing these TAAs and could induce a cytotoxic T-cell-mediated immune response against renal cell cancer. 